\title{
A NOTE ON KARAMATA'S GENERALISED REGULAR VARIATION
}

\author{
R. A. MALLER \\ (Received 13 July 1976; revised 30 March 1977) \\ Communicated by J. Gani
}

\begin{abstract}
In this note, a one sided aspect of regular variation is considered, and some different results which can be obtained by bounding the ratio $f(x \lambda) / f(x)$ above or below are given.
\end{abstract}

In this note we consider some aspects of a one-sided type of regular variation. We let $f$ be a positive measurable function on $[0, \infty)$, and consider the condition

$$
\begin{gathered}
\limsup _{x \rightarrow+\infty} f(x \lambda) / f(x)<+\infty \text { for } \lambda \text { in a set of positive measure } \\
\text { contained in }[1,+\infty) .
\end{gathered}
$$

Later, we show how this condition is related to some used by Karamata and other authors. We make the further assumptions throughout that $f$ is integrable on all intervals $[0, x], x>0$, and that $\int_{0}^{1} u^{k} f(u) d u$ converges for any $k$. These are merely for convenience in the notation, since we are only interested in the asymptotic behaviour of $f$.

One of the important uses of the theory of regular variation is to relate the asymptotic behaviour of the integral $\int_{0}^{x} u^{k} f(u) d u$ to that of $f(x)$ and, in general, we would like to know when

$$
0<\liminf _{x \rightarrow+\infty} \frac{x^{k+1} f(x)}{\int_{0}^{x} u^{k} f(u) d u} \text {, and } \limsup _{x \rightarrow+\infty} \frac{x^{k+1} f(x)}{\int_{0}^{x} u^{k} f(u) d u}<+\infty .
$$


In this note we find conditions under which the two inequalities in (2) hold for any $k$, and hence show that there is an essential dis-symmetry between them. In Lemma 1 below, we see that the right hand inequality in (2) holds whenever (1) holds; but that, for the left hand inequality in (2), we require in addition that $\lim \sup _{\lambda \rightarrow+\infty} \lambda^{k+1} \lim _{\inf _{x \rightarrow+\infty}} f(x \lambda) / f(x)=+\infty$.

Our main result is:

Theorem 1. Suppose (1) holds, and $\int_{0}^{\infty} u^{k} f(u) d u=+\infty$. Then

$$
\liminf _{x \rightarrow+\infty} \frac{x^{k+1} f(x)}{\int_{0}^{x} u^{k} f(u) d u}>0
$$

holds if

$$
\limsup _{\lambda \rightarrow+\infty} \lambda^{k+1} \liminf _{x \rightarrow+\infty} f(x \lambda) / f(x)=+\infty
$$

while

$$
\liminf _{x \rightarrow+\infty} \frac{x^{k+1} f(x)}{\int_{0}^{x} u^{k} f(u) d u}>0
$$

implies

$$
\lim _{\lambda \rightarrow+\infty} \lambda^{k+1} \liminf _{x \rightarrow+\infty} f(x \lambda) / f(x)=+\infty .
$$

The proof of Theorem 1 is accomplished through a series of lemmas, some of which are of independent interest.

- Lemma 1. If (1) holds, then for any $k$,

$$
\limsup _{x \rightarrow+\infty} \frac{x^{k+1} f(x)}{\int_{0}^{x} u^{k} f(u) d u}=c_{k}<+\infty .
$$

Proof of Lemma 1. Suppose not; then for some $k$ there is a sequence $x_{n} \rightarrow+\infty$ for which

$$
\begin{aligned}
0 & =\liminf _{n \rightarrow+\infty} \int_{0}^{x_{n}} u^{k} f(u) d u / x_{n}^{k+1} f\left(x_{n}\right)=\liminf _{n \rightarrow+\infty} \int_{0}^{1} u^{k} f\left(u x_{n}\right) d u / f\left(x_{n}\right) \\
& \geqq \int_{0}^{1} u^{k} \liminf _{n \rightarrow+\infty} f\left(u x_{n}\right) / f\left(x_{n}\right) d u,
\end{aligned}
$$


by Fatou's Lemma. This means that $\liminf _{n \rightarrow+\infty} f\left(\lambda x_{n}\right) / f\left(x_{n}\right)=0$ a.e. $\lambda \in[0,1)$, $\liminf _{x \rightarrow+\infty} f(\lambda x) / f(x)=0$ a.e. $\lambda \in[0,1)$, and limsup $\sup _{x \rightarrow+\infty} f(x \lambda) / f(x)=+\infty$ a.e. $\lambda>1$, contradicting (1).

Lemma 2. If (1) holds, there are constants $N, x_{0}$ and $\lambda_{0} \geqq 1$ for which $f(x \lambda) / f(x) \leqq \lambda^{N}$, whenever $\lambda \geqq \lambda_{0}$ and $x \geqq x_{0}$.

Proof OF LemMa 2. Let $\phi(x)=\log f\left(e^{x}\right)$, let $\psi(\lambda)=$ $\limsup \sup _{x \rightarrow+\infty}[\phi(x+\lambda)-\phi(x)]$, and for integers $n \geqq 1$ define the sets

$$
S_{n}=\{\lambda \geqq 0: \psi(\lambda) \leqq n \lambda\}
$$

so that

$$
\bigcup_{n \geqq 1} S_{n}=\{\lambda \geqq 0: \psi(\lambda)<+\infty\},
$$

and hence by (1), $S_{m,}$ has positive measure for some $n_{0} \geqq 1$. Clearly, if $\lambda_{1}, \lambda_{2} \in S_{n_{1}}$,

$$
\psi\left(\lambda_{1}+\lambda_{2}\right) \leqq \psi\left(\lambda_{1}\right)+\psi\left(\lambda_{2}\right) \leqq n_{0}\left(\lambda_{1}+\lambda_{2}\right) .
$$

So $\lambda_{1}+\lambda_{2} \in S_{n_{0}}$, and by a well known result of Steinhaus, these properties mean that $S_{n_{0}}$ contains an interval $[A, \infty)$. Recalling the definition of $\phi$, this means that

$$
\limsup _{x \rightarrow+\infty} f(x \lambda) / f(x) \leqq \lambda^{n_{0}}, \text { for } \lambda \geqq e^{A} .
$$

This inequality, apparently stronger than (1), was imposed in place of (1) in the original version of this paper; a referee showed me the above argument, for which I am grateful. Now to derive the uniform bound of the lemma, we trivially modify Letac (1970). Define $\phi$ as above, and for $\varepsilon>0$ let

$$
T_{n}=\left\{\lambda \geqq A: \phi(x+\lambda)-\phi(x) \leqq\left(n_{0}+\varepsilon\right) \lambda, \text { for } x \geqq n\right\} .
$$

Then $\bigcup_{n \geqq 1} T_{n}=[A, \infty)$ and so for some $n_{1}, T_{n}$ has positive measure, and is easily seen, like $S_{n_{0}}$, to be a semigroup. Hence $T_{n_{1}}$ contains an interval $[B, \infty)$. This means that $f(x \lambda) / f(x) \leqq \lambda^{n_{0}+e} \equiv \lambda^{N}$ for $\lambda \geqq \lambda_{0}=e^{B}$ and $x \geqq x_{0}=e^{n}$. This completes the proof of Lemma 2.

Lemma 3. If (1) holds then

$$
\limsup _{x \rightarrow+\infty} \int_{x_{\lambda, 1}}^{x \lambda} u^{k} f(u) d u / x^{k+1} f(x)=f_{k}(\lambda)<+\infty, \text { for } \lambda \geqq \lambda_{10},
$$

where $\lambda_{0}$ is defined in Lemma 2. 
Proof of Lemma 3. For $\lambda \geqq \lambda_{0}$,

$$
\begin{aligned}
\limsup _{x \rightarrow+\infty} \int_{x_{\lambda_{0}}}^{x_{\lambda}} u^{k} f(u) d u / x^{k+1} f(x) & =\limsup _{x \rightarrow+\infty} \int_{\lambda_{0}}^{\lambda} u^{k} f(u x) d u / f(x) \\
& \leqq \limsup _{x \rightarrow+\infty} \sup _{\lambda_{0} \leqq u \leqq \lambda}[f(u x) / f(x)] \int_{\lambda_{0}}^{\lambda} u^{k} d u \\
& \leqq \sup _{\lambda_{1} \leqq u \leqq \lambda} u^{N} \int_{\lambda_{0}}^{\lambda} u^{k} d u<+\infty
\end{aligned}
$$

by using the result of Lemma 2 .

LEMMA 4. If (3) holds and $\liminf _{x \rightarrow+x}\left(x^{k+1} f(x) / \int_{0}^{x} u^{k} f(u) d u\right)=b>0$ then

$$
\liminf _{x \rightarrow+x} f(x \lambda) / f(x) \geqq b c_{k}^{-1} \lambda^{b-k-1}, \quad \text { for } \lambda \geqq 1 \text {. }
$$

Proof of Lemma 4. For $\lambda>1, \varepsilon>0$ and $x \geqq x_{0}(\varepsilon)$,

$$
\begin{aligned}
\frac{\int_{0}^{x \lambda} u^{k} f(u) d u}{\int_{0}^{x} u^{k} f(u) d u} & =\exp \int_{x}^{x \lambda} \frac{u^{k+1} f(u) u^{-1} d u}{\int_{0}^{u} y^{k} f(y) d y} \\
& \geqq \exp \left[(b-\varepsilon) \int_{x}^{x \lambda} u^{-1} d u\right] \\
& =\lambda^{b-\varepsilon},
\end{aligned}
$$

(c.f. Matuszewska (1962) page 336). Hence, by what we have just proved and the definitions of $b$ and $c_{k}$,

$$
\begin{aligned}
& \lambda^{b-\varepsilon} \int_{0}^{x} u^{k} f(u) d u \leqq \int_{0}^{x \lambda} u^{k} f(u) d u \\
& (b-\varepsilon) \int_{0}^{x \lambda} u^{k} f(u) d u \leqq \lambda^{k+1} x^{k+1} f(x \lambda) \\
& x^{k+1} f(x) \leqq\left(c_{k}+\varepsilon\right) \int_{0}^{x} u^{k} f(u) d u
\end{aligned}
$$

for $x \geqq x_{1}(\varepsilon, \lambda)$. This means that

$$
\lambda^{b-\varepsilon} x^{k+1} f(x) \leqq\left(c_{k}+\varepsilon\right)(b-\varepsilon)^{-1} \lambda^{k+1} x^{k+1} f(x \lambda),
$$

leading to the desired result. 
Lemma 5. Let (1) hold, suppose $\int_{0}^{\infty} u^{k} f(u) d u=+\infty$, and let $h(\lambda)=$ $\liminf _{x \rightarrow+\infty} f(x \lambda) / f(x)$, for $\lambda>1$. Then

$$
\liminf _{x \rightarrow+\infty} \frac{x^{k+1} f(x)}{\int_{0}^{x} u^{k} f(u) d u} \geqq \frac{\lambda^{k+1} h(\lambda)-\lambda_{0}^{c_{k}}}{f_{k}(\lambda)} \text { for } \lambda \geqq \lambda_{0},
$$

where $c_{k}$ is defined in (3) and $\lambda_{0}$ and $f_{k}(\lambda)$ in Lemmas 2 and 3.

Proof. Take $\lambda>\lambda_{0}$. If $h(\lambda)=0$ there is nothing to prove and so take $h(\lambda)>0$. Given $\varepsilon>0$ with $h(\lambda)-\varepsilon>0$ take $x_{0}=x_{0}(\varepsilon, \lambda)$ so that $f(x \lambda) / f(x) \geqq h(\lambda)-\varepsilon$, and $\int_{x \lambda_{0}}^{x \lambda} u^{k} f(u) d u / x^{k+1} f(x) \leqq f_{k}(\lambda)+\varepsilon$, for $x \geqq x_{0} ;$ the latter can be accomplished by Lemma 3. Now

$$
\begin{aligned}
\int_{0}^{x} u^{k} f(u) d u= & \int_{0}^{x_{0}} u^{k} f(u) d u+\int_{x_{0}}^{x} u^{k} f(u) d u \\
\leqq & o\left(\int_{0}^{x} u^{k} f(u) d u\right)+\int_{x_{0}}^{x} u^{k} f(u \lambda) d u /[h(\lambda)-\varepsilon] \\
\leqq & o\left(\int_{0}^{x} u^{k} f(u) d u\right)+\lambda^{-k-1} \int_{0}^{x \lambda} u^{k} f(u) d u /[h(\lambda)-\varepsilon] \\
\leqq & o\left(\int_{0}^{x} u^{k} f(u) d u\right)+\lambda^{-k-1} \int_{0}^{x_{0}} u^{k} f(u) d u /[h(\lambda)-\varepsilon] \\
& +\lambda^{-k-1} \int_{x \lambda_{0}}^{x \lambda} u^{k} f(u) d u /[h(\lambda)-\varepsilon] \\
\leqq & o\left(\int_{0}^{x} u^{k} f(u) d u\right)+\lambda^{-k-1}\left(\lambda_{0}^{c^{k}}+\varepsilon\right) \int_{0}^{x} u^{k} f(u) d u /[h(\lambda)-\varepsilon] \\
& +\lambda^{-k-1}\left[f_{k}(\lambda)+\varepsilon\right] x^{k+1} f(x) /[h(\lambda)-\varepsilon] .
\end{aligned}
$$

where we have used also the fact that

$$
\limsup _{x \rightarrow+\infty} \frac{\int_{0}^{x \lambda} u^{k} f(u) d u}{\int_{0}^{x} u^{k} f(u) d u} \leqq \lambda^{c_{k}}
$$

for $\lambda \geqq 1$, which we will prove later. Continuing, we get

$$
\lambda^{k+1}[h(\lambda)-\varepsilon] \leqq o(1)+\lambda_{0}^{c_{k}}+\varepsilon+\left[f_{k}(\lambda)+\varepsilon\right] \frac{x^{k+1} f(x)}{\int_{0}^{x} u^{k} f(u) d u},
$$

which leads to the required result. To complete the proof, note that for $\lambda>1$, $\varepsilon>0$ and $x \geqq x_{0}(\varepsilon, \lambda)$, by Lemma 1 , 


$$
\begin{aligned}
\frac{\int_{0}^{x \lambda} u^{k} f(u) d u}{\int_{0}^{x} u^{k} f(u) d u} & =\exp \int_{x}^{x \lambda} \frac{u^{k+1} f(u) u^{-1} d u}{\int_{0}^{u} y^{k} f(y) d y} \\
& \leqq \exp \left[\left(c_{k}+\varepsilon\right) \int_{x}^{x \lambda} u^{-1} d u\right] \\
& =\lambda^{c_{k}+\varepsilon}
\end{aligned}
$$

which is what we needed.

Proof of TheORem 1. If $\liminf _{x \rightarrow+x}\left(x^{k+1} f(x) / \int_{0}^{x} u^{k} f(u) d u\right)=b>0$, then by Lemma 4 , for $\lambda \geqq 1$,

$$
\lambda^{k+1} \liminf _{x \rightarrow+\infty} f(x \lambda) / f(x) \geqq b c_{k}^{-1} \lambda^{b} \rightarrow+\infty \text { as } \quad \lambda \rightarrow+\infty
$$

Conversely, if limsup $\operatorname{sum}_{\lambda \rightarrow+x} \lambda^{k+1} \liminf _{x \rightarrow+\infty} f(x \lambda) / f(x)=+\infty$, there is a $\lambda_{1}>\lambda_{0}$ for which $\lambda_{1}^{k+1} \lim \inf _{x \rightarrow+\infty} f\left(x \lambda_{1}\right) / f(x)>2 \lambda_{0}^{c_{k}}$, where $c_{k}$ is defined in Lemma 1 and $\lambda_{0}$ in Lemma 2. From Lemma 5, taking $\lambda=\lambda_{1}$, we see that $\liminf \operatorname{in}_{x \rightarrow+\infty}\left(x^{k+1} f(x) / \int_{0}^{x} u^{k} f(u) d u\right)>\lambda_{0}^{c_{k}} / f_{k}\left(\lambda_{1}\right)>0$.

REMARKS. (i) When $\int_{0}^{\infty} u^{k} f(u) d u<+\infty$, similar methods can be used to consider $\int_{x}^{\infty} u^{k} f(u) d u$, instead of $\int_{0}^{x} u^{k} f(u) d u$.

(ii) When $f$ is nonincreasing, (1) holds trivially, and Lemmas 1, 2 and 3 are obvious. Lemma 4 was proved, in essence, by Feller (1969) under this restriction.

(iii) A function $f$, positive and measurable on $[A, \infty)$ for some $A>0$, was said by Karamata to be $R-0$ varying if for some $m \leqq 1, M \geqq 1, a>1$,

$$
m \leqq f(x \lambda) / f(x) \leqq M, \text { for } 1 \leqq \lambda \leqq a \text { and } x \geqq A .
$$

An $R-0$ varying function $f$ is $S-0$ varying if for some $c \in(0,1]$ and $C \geqq 1$,

$$
c \leqq \liminf _{x \rightarrow+x} f(x \lambda) / f(x) \leqq \limsup _{x \rightarrow+x} f(x \lambda) / f(x) \leqq C, \quad \text { for } \lambda \geqq 1 \text {. }
$$

The concepts of $R-0$ and $S-0$ variation have recently been reviewed and extended by Seneta (1976). He showed (page 94) that if $f$ is $R-0$ varying, then (2) holds for any $k>h-1$, where $h$ is a constant depending on the values of $a$ and $m$ in (4).

Functions satisfying (1) are related to the $\Delta_{\lambda}$-functions of Matuszewska (1962), who assumed that the inequality in (1) holds for all $\lambda \geqq 1$.

(iv) The right hand side of (4) implies (1), and if we assume it, we can rewrite Theorem 1 as: 
THEOREM 2. Assume $f(x \lambda) / f(x) \leqq M<+\infty$ for $\lambda \in[1, a]$ for some $a>$ 1 and $M \geqq 1$ whenever $x \geqq A$, and suppose $\int_{0}^{\infty} u^{k} f(u) d u=+\infty$. Then $\liminf \sin _{x \rightarrow+\infty}\left(x^{k+1} f(x) / \int_{0}^{x} u^{k} f(u) d u\right)=0$ if and only if $\liminf _{x \rightarrow+\infty} f(x \lambda) / f(x) \leqq$ $\lambda^{-k-1}$, for $\lambda \geqq 1$.

Proof of Theorem 2. If (4) holds, and $\lambda \in\left[a^{p-1}, a^{p}\right]$ for some $p>1$, then as in Lemma A.1 of Seneta (1976 page 92), we can deduce that, for $x \geqq A, f(x \lambda) / f(x) \leqq M^{p}$. (Thus in fact $f$ is a $\Delta_{\lambda}$-function.) Furthermore, the conclusion of Lemma 3 now holds with $\lambda_{0}=1$. The conclusion of Theorem 2 is just the contrapositive of the conclusions of Lemmas 4 and 5 , when $\lambda_{0}$ is put equal to 1.

To conclude our discussion we remark that one can always say something about the ratio $f(x \lambda) / f(x)$ in the following sense:

Leмma 6. Let $f$ be positive, measurable and integrable on $[a, \infty)$. Then: if $\int_{a}^{x} f(u) d u=+\infty$, we have $\lim \sup _{x \rightarrow+\infty} f(x \lambda) / f(x) \geqq \lambda^{-1}$, for $\lambda \geqq 1$; if $\int_{a}^{x} f(u) d u<+\infty$, we have $\liminf \operatorname{for}_{x \rightarrow+\infty} f(x \dot{\lambda}) / f(x) \leqq \lambda^{-1}$, for $\lambda \geqq 1$.

ProOF. Suppose $\int_{a}^{\infty} f(u) d u=+\infty$ and let $g(\lambda)=\limsup _{x \rightarrow+\infty} f(x \lambda) / f(x)$ for $\lambda \geqq 1$. Given $\lambda>1$ and $\varepsilon>0$ choose $x_{0}=x_{0}(\varepsilon, \lambda) \geqq a$, so that $f(x \lambda) \leqq$ $[g(\lambda)+\varepsilon] f(x)$ whenever $x \geqq x_{0}$. Then for $x \geqq x_{0}$,

$$
\begin{aligned}
\int_{a}^{x} f(u) d u & \leqq \int_{a}^{x \lambda} f(u) d u=\lambda \int_{a / \lambda}^{x} f(u \lambda) d u \\
& =\lambda \int_{a / \lambda}^{x_{1 \prime}} f(u \lambda) d u+\lambda \int_{x_{0}}^{x} f(u \lambda) d u \\
& \leqq o\left(\int_{a}^{x} f(u) d u\right)+\lambda[g(\lambda)+\varepsilon] \int_{x_{0}}^{x} f(u) d u \\
& \leqq o\left(\int_{a}^{x} f(u) d u\right)+\lambda[g(\lambda)+\varepsilon] \int_{a}^{x} f(u) d u,
\end{aligned}
$$

so $g(\lambda) \geqq \lambda^{-1}$, as required. The other proof is similar. Using this lemma, we see that Theorem 2 holds even when $\int_{0}^{x} u^{k} f(u) d u<+\infty$.

A result related to Lemma 6 is given by Seneta (1976) page 99: if $f$ is positive on $[A, \infty)$, bounded on finite intervals sufficiently far, and $0<$ $\lim \sup _{x \rightarrow+\infty} f(x \lambda) / f(x)<+\infty$, then $\lim \sup _{x \rightarrow+\infty} f(x \lambda) / f(x) \geqq \lambda^{\rho}$ for $\lambda \geqq 1$ for some $\rho$.

\section{Acknowledgements}

I am grateful to Dr E. Seneta for many discussions on the theory of regular variation, for referring me to the work of Letac (1970) and for 
suggesting the modification of Letac's method used in Lemma 2. I am also grateful for a CSIRO Division of Mathematics \& Statistics Postgraduate Studentship.

\section{References}

Feller, W. (1969), 'One sided analogues of Karamata's regular variation', Enseign. Math. 15, 107-121.

Letac, G. (1970), 'On slow variation', Proc. Amer. Math. Soc. 24, 745-746.

Matuszewska, W. (1962), 'Regularly increasing functions in connection with the theory of $L^{* \infty}$ spaces', Studia Math. 21, 317-344.

Seneta, E. (1976), Regularly Varying Functions (Lecture Notes in Mathematics, 508 Springer, Berlin).

Australian National University, and

C.S.I.R.O. Division of Mathematics and Statistics, PO Box 310 South Melbourne 3205, Australia. 